\title{
Inducibility of atrial fibrillation during electrophysiologic evaluation is associated with increased dispersion of atrial refractoriness
}

\author{
Mario Oliveira ${ }^{\text {a,*}}$, M. Nogueira da Silva ${ }^{\text {a }}$, Ana T. Timoteo ${ }^{a}$, Joana Feliciano ${ }^{\text {a }}$, Lidia Sousa ${ }^{\text {a }}$, \\ Sofia Santos ${ }^{\mathrm{a}}$, Luis Silva-Carvalho ${ }^{\mathrm{b}}$, Rui Ferreira ${ }^{\mathrm{a}}$

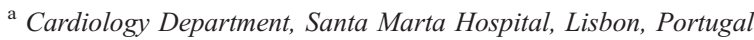 \\ ${ }^{\mathrm{b}}$ Institute of Physiology, Faculty of Medicine of Lisbon, Portugal
}

Received 7 September 2007; received in revised form 20 March 2008; accepted 23 April 2008

Available online 3 August 2008

\begin{abstract}
The impact of atrial dispersion of refractoriness (Disp_A) in the inducibility and maintenance of atrial fibrillation (AF) has not been fully resolved. Aim: To study the Disp_A and the vulnerability (A_Vuln) for the induction of self-limited ( $<60$ s) and sustained episodes of AF. Methods and results: Forty-seven patients with paroxysmal AF (PAF): 29 patients without structural heart disease and 18 with hypertensive heart disease. Atrial effective refractory period (ERP) was assessed at five sites - right atrial appendage and low lateral right atrium, high interatrial septum, proximal and distal coronary sinus. We compared three groups: group A - AF not inducible ( $n=13)$; group B - AF inducible, self-limited $(n=18)$; group C - AF inducible, sustained $(n=16)$. Age, lone AF, hypertension, left atrial and left ventricular (LV) dimensions, LV systolic function, duration of AF history, atrial flutter/tachycardia, previous antiarrhythmics, and Disp_A were analysed with logistic regression to determine association with A_Vuln for AF inducibility. The ERP at different sites showed no differences among the groups. Group A had a lower Disp_A compared to group B ( $47 \pm 20 \mathrm{~ms}$ vs $82 \pm 65 \mathrm{~ms} ; p=0.002)$, and when compared to group C (47 $\pm 20 \mathrm{~ms}$ vs $80 \pm 55 \mathrm{~ms} ; p=0.008$ ). There was no significant difference in Disp_A between groups B and C. By means of multivariate regression analysis, the only predictor of A_Vuln was Disp_A $(p=0.04)$. Conclusion: In patients with PAF, increased Disp_A represents an electrophysiological marker of A_Vuln. Inducibility of both self-limited and sustained episodes of AF is associated with similar values of Disp_A. These findings suggest that the maintenance of AF is influenced by additional factors.
\end{abstract}

(C) 2008 Elsevier Ireland Ltd. All rights reserved.

Keywords: Dispersion of refractoriness; Atrial vulnerability; Non-sustained and sustained atrial fibrillation

\section{Introduction}

Atrial fibrillation (AF) is the most common sustained cardiac arrhythmia in the general practice setting. Its prevalence increases with age, from $0.4 \%$ in the general population to more than $5 \%$ over the age of $65[1,2]$, and it is recognized as a potentially dangerous arrhythmia, with impact on both life expectancy and quality of life $[3,4]$. AF remains a considerable clinical challenge, in part due to our

\footnotetext{
* Corresponding author.

E-mail address: m.martinsoliveira@gmail.com (M. Oliveira).
}

limitations in understanding the electrophysiological mechanisms underlying the condition. Despite the amount of recent information on management and therapeutic strategies on $\mathrm{AF}$, we still have limited knowledge regarding the mechanisms of arrhythmia recurrence and progression to sustained AF. In fact, paroxysmal AF, defined as recurrent, self-terminating within 7 days of onset, progresses to persistent $\mathrm{AF}$ in over $18 \%$ of patients, even if there is no sign of underlying structural heart disease $[5,6]$.

Electrical remodelling of the atrial tissue, which is associated with shortening of the atrial refractory period in a heterogeneous way, is known to be related with atrial vulnerability for the occurrence of spontaneous and inducible 
$\mathrm{AF}$ and to favour the maintenance and perpetuation of the arrhythmia [7]. Patients with inducible AF are at an increased risk of AF recurrence, even after pulmonary vein isolation $[8,9]$. Atrial effective refractory periods (ERP) and its spatial dispersion heterogeneity have been accepted to promote AF re-initiation and to provide a substrate for the re-entry of multiple wavelets to enhance the ability of the disorder to sustaining itself $[10,11]$. Also, an increase in the electrical homogeneity or a decrease in the dispersion of refractoriness may contribute to decrease the number of wavelets and lead to the AF termination [12]. Studies have shown that spatial dispersion of refractoriness is involved in the maintenance of AF $[13,14]$. Increased dispersion of atrial refractoriness and shortening of wavelength have been also correlated with initiation and maintenance of $\mathrm{AF}$ after its induction in a pacing-induced model of AF in the pig [15]. Nevertheless, there is lack of data concerning the impact of the degree of the non-uniformity of ERP on the vulnerability for the inducibility and for the persistence of AF among humans.

In the present study, we investigated whether the dispersion of atrial refractoriness influences the vulnerability for the induction of $\mathrm{AF}$ in patients with paroxysmal AF (PAF). Additionally, we evaluated the relationship between the magnitude of atrial refractoriness dispersion and inducibility of self-limited and self-sustained AF.

\section{Methods}

\subsection{Patient population}

The study consisted of 47 patients referred to our institution, with $\geq 1$ year duration of clinical history of PAF, despite antiarrhythmic therapy. PAF was documented with electrocardiograms and/or Holter recordings. Patients with evidence of sick sinus syndrome, failure to remain in stable sinus rhythm while in-hospital monitoring before the electrophysiological study (EPS), permanent pacemaker implanted, bronchopulmonary disease and pregnancy or thyroid dysfunction were not included in the study. Prior to the EPS, all antiarrhythmic drugs were withdrawn for at least 5 half-life times. Patients under amiodarone stopped treatment 2 months before the EPS. The study protocol was approved by the local ethics. All subjects were required to give written informed consent.

The study protocol was performed according to the ethical guidelines of the Declaration of Helsinki.

\subsection{Electrophysiological protocol}

All patients underwent EPS in a non-sedated postabsorptive state. No serum electrolyte disturbances were found.

Electrical programmed stimulation and recording of electrograms were performed by using $6 \mathrm{~F}$ catheter electrodes inserted percutaneously into the femoral and internal jugular veins. A quadripolar electrode catheter (2-mm-spaced; Daig
Co) was positioned in the right atrial appendage (RAA), and moved to the low right posterolateral atrium (LRA) and high interatrial septum (IAS), a second quadripolar electrode catheter (2-mm-spaced; Daig Co) was inserted into the His bundle area (HBE), and a 2-mm-spaced decapolar electrode catheter (Daig Co) was advanced into the coronary sinus (CS). All bipolar electrograms were recorded using a multichannel electrophysiological recorder (Bard Lab System) with a frequency response of $50-500 \mathrm{~Hz}$ onto optical disks for later analysis. Twelve-lead surface ECGs were also simultaneously recorded. Hard copies of the electrograms were printed at a recording speed of $100 \mathrm{~mm} / \mathrm{s}$.

As a measure of local refractoriness, ERP were assessed in each patient at five different sites (RAA, LRA, IAS, proximal and distal CS). Under stable conditions, a programmed electrical stimulation using a single premature stimulus (S2) was delivered, while pacing continuously at a basic drive cycle length of $600 \mathrm{~ms}$. Stimulation was performed with impulses of $2 \mathrm{~ms}$ duration at twice the diastolic threshold. A premature beat was introduced in late diastole, beginning at a coupling interval of $100 \mathrm{~ms}$ less than the basic cycle length. The coupling interval of the premature stimulation was decreased by $10 \mathrm{~ms}$ steps until the ERP was reached. The ERP were taken as the longest S1-S2 intervals that failed to initiate a propagation response. Dispersion of refractoriness was obtained in all patients as the difference between the longest and the shortest ERP at the five stimulation sites.

All patients underwent programmed bipolar stimulation (drive-train cycle length of $600 \mathrm{~ms}$ using S2-S3 extrastimuli) and incremental pacing protocols (short-term of burst pacing range from 600 to $300 \mathrm{~ms}$ ) during sinus rhythm by pacing from the distal electrode pairs positioned at the RAA and distal CS catheters. AF was defined as a rapid atrial rhythm (rate $>350$ beats $/ \mathrm{min}$ ) characterized by variability of the beat-to-beat cycle length, polarity, configuration and amplitude of the recorded atrial electrograms and lasting more than 5 cycles [16]. The concept of atrial vulnerability was based on the ability to induce AF with 1-2 extra-stimuli or with incremental atrial pacing during electrical stimulation from the RAA or distal CS. If AF was induced, an external electrical cardioversion was performed after $\geq 5 \mathrm{~min}$ of continuous AF without spontaneous termination. In patients requiring external cardioversion, a maximum of 3 shocks was delivered. The patients were separated into group A AF not inducible, group B - AF inducible, self-limited $(<60 \mathrm{~s})$, and group $\mathrm{C}-\mathrm{AF}$ inducible, self-sustained, terminated by therapeutic intervention.

\subsection{Statistical analysis}

The results are presented as mean value \pm standard deviation. Categorical variables are expressed as frequencies and percentages. Student's $t$ test and repeated ANOVA were utilised for the analysis of continuous variables (overall comparison). The Chi-square test was used to evaluate the 
Table 1

Clinical characteristics of the patients.

\begin{tabular}{lllll}
\hline Characteristic & $\begin{array}{l}\text { All patients } \\
(n=47)\end{array}$ & $\begin{array}{l}\text { Group A } \\
(n=13)\end{array}$ & $\begin{array}{l}\text { Group B } \\
(n=18)\end{array}$ & $\begin{array}{l}\text { Group C } \\
(n=16)\end{array}$ \\
\hline Age, years & $56 \pm 14$ & $57 \pm 13$ & $54 \pm 15$ & $57 \pm 14$ \\
Male gender & $47 \%$ & $47 \%$ & $50 \%$ & $44 \%$ \\
History of hypertension & $36 \%$ & $38.5 \%$ & $33.3 \%$ & $44 \%$ \\
Number of previous AA & $1.7 \pm 0.8$ & $1.6 \pm 0.9$ & $1.4 \pm 0.8$ & $2.0 \pm 0.5$ \\
LA $\geq 22 \mathrm{~mm} / \mathrm{m}^{2}$ & $36 \%$ & $38.5 \%$ & $38.9 \%$ & $31 \%$ \\
LV hypertrophy & $12.7 \%$ & $15.3 \%$ & $11.1 \%$ & $12.5 \%$ \\
LVEF $<50 \%$ & $11.1 \%$ & $15.4 \%$ & $11.1 \%$ & $6.3 \%$ \\
Duration of AF (years) & $2.3 \pm 1.9$ & $2.0 \pm 1.5$ & $1.9 \pm 2.0$ & $3.0 \pm 2.2$ \\
AFL/AT & $11.1 \%$ & $15.4 \%$ & $5.6 \%$ & $12.5 \%$ \\
\hline
\end{tabular}

$\mathrm{AA}=$ antiarrhythmics; LA =left atrium (M-mode measurements); LV=left ventricle; $\mathrm{LVEF}=$ left ventricular ejection fraction; $\mathrm{AF}=$ atrial fibrillation; $\mathrm{AFL}=$ atrial flutter; $\mathrm{AT}=$ atrial tachycardia.

None of the variables differed significantly between the groups.

differences in categorical variables. Logistic regression analysis was used to assess the relation of variables with atrial vulnerability for the induction of AF. We tested the following variables for all patients: age, sex, diagnosis of lone PAF, history of systemic hypertension, left atrial dimension, left ventricular ejection fraction, presence or absence of left ventricular hypertrophy, duration of clinical paroxysmal AF, number of previous antiarrhythmics, documentation of atrial flutter, and dispersion of atrial ERP. For all tests a value of $p<0.05$ was considered statistically significant. Data were analyzed using GraphPAD Instruments (GraphPad Software, Inc., California, USA).

\section{Results}

\subsection{Patient characteristics}

Forty-seven patients ( $47 \%$ male) with a mean age of $56 \pm$ 14 years (range, 18 to 76 years) were subjected to this study. The average duration of the history of PAF was $2.3 \pm 1.9$ years (median 1 year; range, 1 to 8 years).The population included 29 patients without structural heart disease and 18 with hypertensive heart disease. AF was inducible in $72 \%$ of the patients and non-inducible in $28 \%$. The patients with atrial vulnerability for arrhythmia induction showed self-limited AF in 53\% and self-sustained $\mathrm{AF}$ requiring electric intervention in

Table 2

Effective refractory periods (ERP) measured at five atrial sites.

\begin{tabular}{llll}
\hline ERP (ms) & Group A $(n=13)$ & Group B $(n=18)$ & Group C $(n=16)$ \\
\hline RAA & $216 \pm 24$ & $204 \pm 9$ & $215 \pm 22$ \\
LRA (lateral) & $218 \pm 24$ & $217 \pm 24$ & $210 \pm 14$ \\
IAS (high) & $232 \pm 40$ & $235 \pm 40$ & $220 \pm 15$ \\
pCS & $245 \pm 33^{*}$ & $270 \pm 24^{*}$ & $260 \pm 40^{*}$ \\
dCS & $242 \pm 34^{*}$ & $340 \pm 126^{*}$ & $256 \pm 35^{*}$ \\
\hline
\end{tabular}

Data are expressed as mean $\pm \mathrm{SD} . \mathrm{RAA}=$ right atrial appendage; $\mathrm{LRA}=$ low right atrium; IAS = interatrial septum; $\mathrm{pCS}=$ proximal coronary sinus; $\mathrm{dCS}=$ distal coronary sinus. $p>0.05$ for comparisons between the groups. $* p<0.01$ for comparisons between ERP in the pCS and dCS vs RAA and LRA.
$47 \%$. Group A included 13 patients ( 8 men and 5 women with a mean age of $57 \pm 13$ years). These patients had a $2.0 \pm$ 1.5 years of clinical history of PAF, refractory to $1.6 \pm 0.9$ antiarrhythmic drugs. Group B consisted of 18 patients (11 men and 7 women with a mean age of $54 \pm 15$ years), who had $1.4 \pm 0.8$ years of PAF, refractory to $1.9 \pm 2.0$ antiarrhythmic drugs. Group $\mathrm{C}$ was composed of 16 patients (10 men and 6 women with a mean age of $57 \pm 14$ years), who had $2.0 \pm$ 0.5 years of history of paroxysmal AF episodes, refractory to $3.0 \pm 2.2$ antiarrhythmic drugs. There were no significant differences regarding clinical and echocardiographic data among the three groups (Table 1).
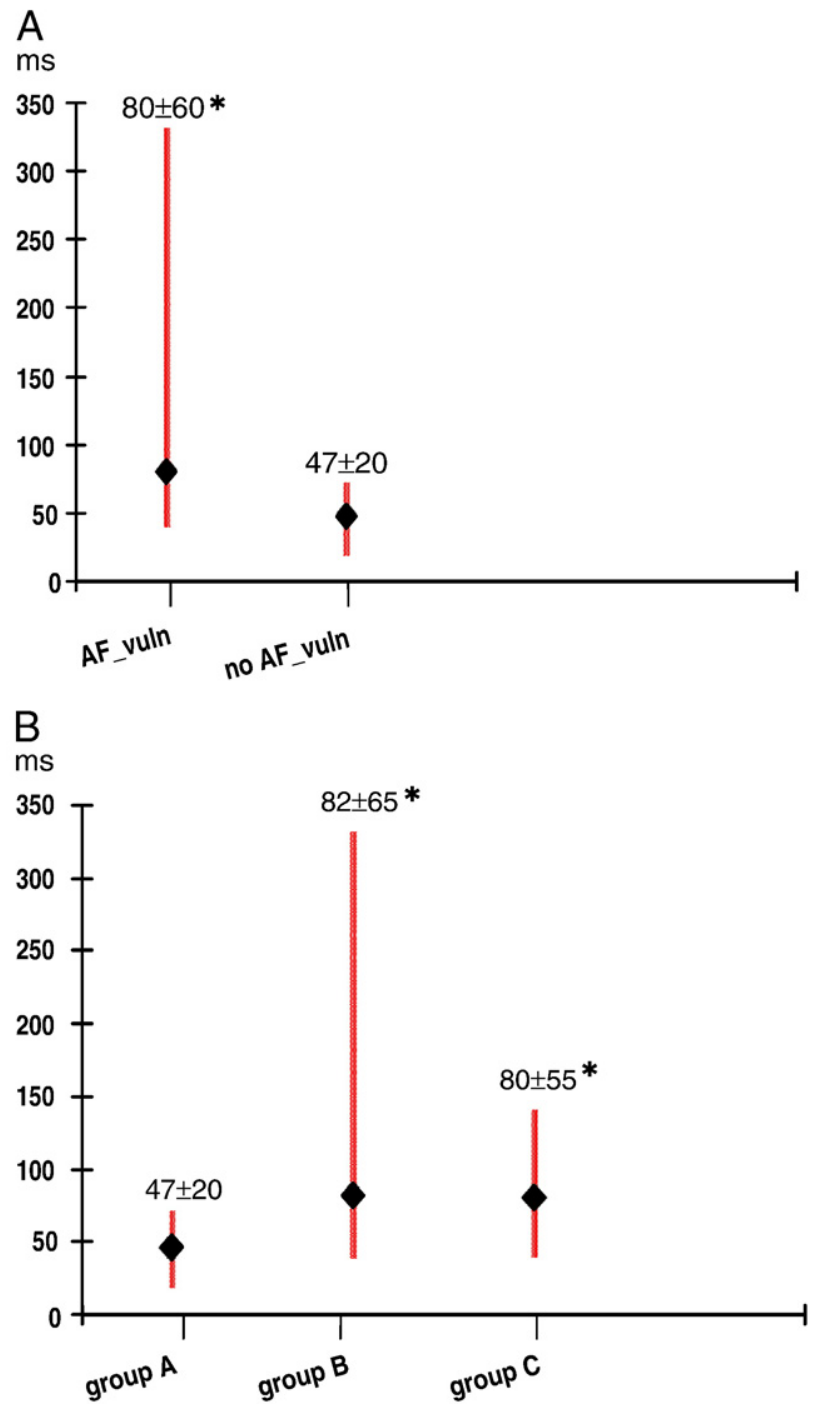

Fig. 1. The line graphs summarize the dispersion of effective atrial refractory periods for all the groups. Data are expressed as mean \pm SD. The lines represent the mean, minimum and maximum values for each group. $\mathrm{A} . \mathrm{AF} \_$vuln $=$patients with vulnerability for $\mathrm{AF}$ induction $(n=34)$; no $\mathrm{AF} \_$vuln=patients without $\mathrm{AF}$ induction $(n=13)$. $\mathrm{B}$. group $\mathrm{A}=\mathrm{AF}$ not inducible $(n=13)$; group $\mathrm{B}=\mathrm{AF}$ inducible, self-limited $(<60 \mathrm{~s})(n=18)$; group $\mathrm{C}=\mathrm{AF}$ inducible, selfsustained $(n=16)$. Asterisks represent statistical significance $\left(\mathrm{AF}_{-}\right.$vuln vs no $\mathrm{AF} \_$vuln, $p=0.01$; group $\mathrm{A}$ vs group $\mathrm{B}, p=0.002$; group $\mathrm{A}$ vs group $\mathrm{C}$, $p=0.008$ ). 


\subsection{Atrial refractoriness}

ERP values measured at the CS (proximal and distal) were significantly higher when compared with the other evaluated sites (Table 2). The mean ERP increased progressively from the RAA, LRA, and IAS to the proximal and distal CS $(212 \pm 18 \mathrm{~ms}, 213 \pm 20 \mathrm{~ms}, 228 \pm 31 \mathrm{~ms}, 256 \pm$ $33 \mathrm{~ms}$ and $273 \pm 73 \mathrm{~ms}$ respectively; ERP at the RAA vs distal $C S, p<0.01)$. There were no significant differences among the ERP values measured in the three groups of patients at any of the five sites that were assessed (Table 2). However, dispersion of ERP was significantly higher in the group of 34 patients who had atrial vulnerability for the induction of AF compared with those who remained in sinus rhythm during the EPS $(80 \pm 60 \mathrm{~ms}$ vs $47 \pm 20 \mathrm{~ms} ; p=0.01)$ (Fig. 1). Moreover, the group A had a significant lower dispersion of the mean ERP compared to the group B (47 \pm $20 \mathrm{~ms}$ vs $82 \pm 65 \mathrm{~ms} ; p=0.002)$ and when compared to the group C $(47 \pm 20 \mathrm{~ms}$ vs $80 \pm 55 \mathrm{~ms} ; p=0.008)$. There was no significant difference in dispersion of ERP between the patients with AF lasting $\leq 60 \mathrm{~s}$ and those with self-sustained AF (Fig. 1). By means of multivariate logistic regression, the only predictor of atrial vulnerability for the induction of AF was dispersion of atrial ERP $(p=0 \cdot 04)$.

\section{Discussion}

\subsection{Major findings}

This study was designed to evaluate the impact of the degree of dispersion of atrial ERP on the vulnerability for the induction and maintenance of AF. The results have demonstrated that ERP dispersion values are determinants of atrial vulnerability. Increased dispersion of refractoriness facilitated AF induction, but the ability to sustain AF may be influenced by other factors in addition to the degree of the non-uniformity of local ERP. In fact, despite a greater dispersion of refractoriness in both groups with inducible $\mathrm{AF}$, the ERP dispersion was similar in patients with inducibility of self-limited $\mathrm{AF}$ and in those patients who have induction of AF lasting $\geq 5 \mathrm{~min}$. This suggests that the electrophysiological substrate that creates the conditions for the maintenance of AF is more complex, probably resulting from the combination of different underlying mechanisms in addition to the magnitude of atrial refractoriness dispersion. It is more likely that multiple variables, including the effects of autonomic nervous system, catecholamines, presence of stretched segments of the atria, ischemia and electrolyte imbalance, conduction abnormalities and other electrophysiological characteristics, contribute to the maintenance of AF.

\subsection{Atrial refractory periods}

Shortening of the atrial ERP has been reported as one of the main underlying electrophysiological changes in patients with sustained AF [17]. Nevertheless, in our study the ERP measurements at different sites where not different between groups. Previous studies have demonstrated that AF lead to a decrease in atrial ERP, without a significant change in conduction velocity $[18,19]$. A shorter ERP can create a shorter wavelength $(\mathrm{ERP} \times$ conduction velocity), which significantly contributes to the maintenance of AF [20]. In our study, the ERP was gradually prolonged from the right to the left side, with higher determinations in the proximal CS and distal CS when compared with the RAA, LRA and IAS. Those patients with induction of AF showed a larger dispersion of refractoriness due to a marked difference between ERP at the right atrium and those obtained along the CS. These findings are in accordance with other authors, who reported shorter ERP in the high right atrium when compared with distal CS in patients with AF [7,21]. This may be explained by a non-uniform distribution of vagal nerve endings, which seems to cause greater changes in refractory period in the RAA than in left atrium [22].

It is widely accepted that heterogeneity of electrophysiological properties may play a major role in favouring re-entry waves, and hence the initiation of AF $[13,23]$. Experimental studies also suggested that ERP dispersion is an important factor in determining the ability to sustain AF [24,25]. Our results showed that dispersion of refractoriness is a suitable indicator of atrial vulnerability for the induction of AF. However, dispersion of atrial refractoriness was not significantly increased in patients with self-sustained AF when compared with the group with inducible non-sustained AF.

\subsection{Self-limited vs self-sustained atrial fibrillation}

AF is generally considered to be maintained by multiple re-entrant wavelets of excitation that propagate in different directions around the atrial myocardium [26,27]. The maintenance of AF seems to depend on the presence of a sufficient number of small wavefronts while undergoing fractionation, collisions and coalescence over the atrial surface. To allow multiple re-entrant wavelets to propagate resulting in a self-perpetuating activity, a critical mass of excitable atrial tissue must exist [28]. Re-entry within the atria is associated with shortening of the ERP with increased dispersion of refractoriness, thereby providing a substrate for initiation of AF. The concept of dispersion of refractoriness is based on the non-uniformity of local atrial refractory periods. This results in the coexistence of regions of the atria with relatively short ERP in close proximity to areas with much longer ERP, instead of a gradual transition. Also, reentrant wavelets must never encounter refractory tissue left over by a previous wavelet, otherwise the wavelets will be extinguished and the arrhythmia will not be sustained. Thus, non-uniform changes in refractoriness are associated with an increased frequency of induction of AF. However, when the dispersion of refractoriness is too large, re-entrant wavelets may be extinguished due to a slower recovery of adjacent 
atrial myocardium. If this tissue cannot recover before the wavelet arrives, the critical number of existing wavelets, essential for the AF maintenance, will not be achieved. Moreover, a larger number of circulating wavelets can exist on the surface of larger atria. So, to accommodate the maximum number of wavelets in a constant area, several factors including atrial size, velocity of conduction and dispersion of atrial refractory periods need to be adequately combined.

Recently, in human studies, the presence of shorter ERP in the pulmonary veins, when compared to left atrial refractoriness, was considered to provide a favourable milieu for the initiation of AF and possibly to sustain fibrillatory activity $[29,30]$. In addition, experimental evidence suggests that certain cases of $\mathrm{AF}$ are maintained by small re-entrant dominant frequency sources (rotors) [31,32]. Despite different concepts to explain the perpetuation of $\mathrm{AF}$, dispersion of atrial refractoriness has been consistently associated with vulnerability to the initiation and maintenance of AF.

\subsection{Study limitations}

First, the lack of a control group without history of AF makes the comparison of the dispersion of ERP between our patients and a population without the arrhythmia impossible. However, the aim of this study was to assess the relationship between the intensity of atrial refractoriness dispersion and the vulnerability for the induction of self-limited and selfsustained AF in a population with PAF. Second, the protocol did not include measurements of the ERP from the pulmonary veins and different left atrial sites. Therefore, it is not possible to compare ERP to the right atrium and pulmonary veins in this population. Nevertheless, in previous studies, the distal CS ERP was accepted as reflecting the ERP of the local left atrial tissue [21,33]. Finally, although the number of subjects included in the study allowed for the identification of significant differences in the dispersion of atrial refractoriness, the resulting sample was relatively small, representing only a subpopulation of patients with PAF and absent or minimal structural heart disease. Thus, further studies in a larger group may be needed to confirm these findings.

\section{Conclusions}

Increased atrial ERP dispersion enhances the propensity for the inducibility of AF during electrophysiologic evaluation. Nevertheless, patients with vulnerability for the induction of $\mathrm{AF}$ lasting less than $1 \mathrm{~min}$ and those with inducibility of self-sustained AF had similar and significant increases in atrial dispersion of refractoriness. These results emphasize the importance of the dispersion of ERP as an electrophysiological marker of vulnerability for the induction of AF, and suggest that the maintenance of AF induced during EPS is influenced by additional factors beyond the degree of the non-uniformity of ERP.

\section{References}

[1] Camm AJ. Preface. In: Murgatroyd, FD, Camm, AJ, editors. Nonpharmacological Treatment of Atrial Fibrillation. Armonk, NY: Futura; 1997.

[2] Feinberg WM, Blackshear JL, Laupacis A, et al. Prevalence, age distributions, and gender of patients with atrial fibrillation: analysis and implications. Arch Intern Med 1995;155:469-73.

[3] Benjamin EJ, Wolf PA, D’Agostino RB, et al. Impact of atrial fibrillation on the risk of death. The Framinghan Heart Study. Circulation 1998;98: 946-52.

[4] Van Den Berg MP, Hassink RJ, Tuinenburg AE, Van Sonderen EF, Lefrandt JD, et al. Quality of life in patients with paroxysmal atrial fibrillation and its predictors: importance of the autonomic nervous system. Eur Heart J 2001;22:247-53.

[5] Peters NS, Schilling RJ, Kanagaratnam P, Markides V. Atrial fibrillation: strategies to control, combat, and cure. Lancet 2002;359(9306):593-603.

[6] Kopecky SL, Gersh BJ, McGoon MD, et al. The natural history of lone atrial fibrillation. A population-based study over three decades. N Engl J Med 1987;317:669-74

[7] Soylu M, Demir AD, Özdemir Ö, et al. Increased dispersion of refractoriness in patients with atrial fibrillation in the early postoperative period after coronary artery bypass grafting. J Cardiovasc Electrophysiol January 2003;14:28-31.

[8] Wang L, Yang H, Zhang Y. Recurrence of symptomatic atrial fibrillation after successful catheter ablation of atrioventricular accessory pathways: a multivariate regression analysis. Int J Clin Pract August 2005;59(8):886.

[9] Richter B, Gwechenberger M, Filzmoser P, Marx M, Lercher P, Gossinger HD. Is inducibility of atrial fibrillation after radio frequency ablation really a relevant prognostic factor? Eur Heart J Nov 2006;27(21): 2553-9.

[10] Nattel S, Li D, Yue L. Basic mechanisms of atrial fibrillation-very new insights into very old ideas. Annu Rev Physiol 2000;62:51-77.

[11] Li Zhen, Hertervig Eva, Carlson Jonas, Camilla J, Bertil O, Yuan S. Dispersion of refractoriness in patients with paroxysmal atrial fibrillation. Evaluation with simultaneous endocardial recordings from both atria. J Electrocardiol 2002;35(3):227-34.

[12] Chen J, Wasmund SL, Hamdan MH. Back to the future: the role of the autonomic nervous system in atrial fibrillation. Pacing Clin Electrophysiol 2006;29:413-21.

[13] Misier A, Opthof T, van Hemel N, et al. Increased dispersion of "refractoriness" in patients with idiopathic paroxysmal atrial fibrillation. J Am Coll Cardiol 1992;19:1531-5.

[14] Sato S, Yamauchi S, Schuessler RB, et al. The effect of augmented atrial hypothermia on atrial refractory period, conduction, and atrial flutter/fibrillation in the canine heart. J Thorac Cardiovasc Surg 1992;104:297-306.

[15] Rahme MM, Cotter B, Leistad E, et al. Persistence of atrial fibrillation after its induction-importance of the duration and dispersion of atrial refractoriness and electrical remodeling. J Cardiovasc Pharmacol Ther Apr 1999;4(2):113-20.

[16] Friedman HS, Sinha B, Tun A, et al. Zones of atrial vulnerability. Relationships to basic cycle length. Circulation 1996;94:1456-64.

[17] Yu WC, Chen SA, Lee SH, Tai CT, et al. Tachycardia-induced change of atrial refractory period in humans. Rate dependency and effects of antiarrhythmic drugs. Circulation 1998;97:2331-7.

[18] Daoud EG, Bogum F, Goyal R, Harvey M, Man KC, Strickberger SA, Morady F. Effect of atrial fibrillation on atrial refractoriness in humans. Circulation 1996;94:1600-6.

[19] Goette A, Honeycutt C, Langberg JJ. Electrical remodeling in atria fibrillation: time course and mechanisms. Circulation 1996;94:2968-74.

[20] Byrd GD, Prasad SM, Ripplinger CM, et al. Importance of geometry and refractory period in sustaining atrial fibrillation. Testing the critical mass hypothesis. Circulation 2005;112:I-7 - I-13.

[21] Ishimatsu T, Hayano M, Hirata T, Iliev II, et al. Electrophysiological properties of the left atrium evaluated by coronary sinus pacing in patients with atrial fibrillation. Pacing Clin Electrophysiol Dec 1999;22(12):1739-46. 
[22] Zipes DP, Mihalick MJ, Robbins GT. Effects of selective vagal and stellate ganglion stimulation on atrial refractoriness. Cardiovasc Res 1974;8:647-55.

[23] Li Z, Hertervig E, Yuan S, Yang Y, Lin Z, Olsson BS. Dispersion of atrial repolarization in patients with paroxysmal atrial fibrillation. Europace 2001;3:285-91.

[24] Olgin JE, Sih HJ, Hanish S, Jayachandran JV, et al. Heterogeneous atrial denervation creates substrate for sustained atrial fibrillation. Circulation Dec 8 1998;98(23):2608-14.

[25] Wang J, Liu L, Feng J, Nattel S. Regional and functional factors determining induction and maintenance of atrial fibrillation in dogs. Am J Physiol 1996;271:H148-58.

[26] Allessie MA, Bonke FI, Schopman FJ. Circus movement in rabbit atrial muscle as a mechanism of tachycardia. The "leading circle" concept: a new model of circus movement in cardiac tissue without the involvement of an anatomical obstacle. Circ Res 1977;41:9-18.

[27] Schilling RJ, Kadish AH, Peters N, et al. Endocardial mapping of atrial fibrillation in the human right atrium using a non-contact catheter. Eur Heart J 2000;21:550-64.
[28] Allessie MA, Konings K, Kirchhof CJ, Wijffels M. Electrophysiologic mechanisms of perpetuation of atrial fibrillation. Am J Cardiol 1996;77:10A-23A.

[29] Adragao P, Santos K, Aguiar C, Neves J, Abecassis M, et al. Atrial fibrillation and effective refractory period of the pulmonary vein ostia. Rev Port Cardiol Oct 2002;21(10):1125-34.

[30] Jais P, Hocini M, Macle L, Choi KJ, Deisenhofer I, Weerasooriya R, et al. Distinctive electrophysiological properties of pulmonary veins in patients with atrial fibrillation. Circulation Nov 5 2002;106(19):2479-85.

[31] Mandapati R, Skanes A, Chen J, Berenfeld O, Jalife J. Stable micro reentrant sources as a mechanism of atrial fibrillation in the isolated sheep heart. Circulation 2000;101:194-9.

[32] Haissaguerre M, Hocini M, Sanders P, Takahashi Y, et al. Localized sources maintaining atrial fibrillation organized by prior ablation. Circulation Feb 7 2006;113(5):616-25.

[33] Chen M, Guo GB, Chang HW. Atrial electrophysiological properties evaluated by right and left atrial pacing in patients with or without atrial fibrillation. Jpn Heart J 2002;43:231-40. 